\title{
Vacuum-energy and the angular-size/redshift diagram for milliarcsecond radio sources
}

\author{
J.C. Jackson and Marina Dodgson
}

Department of Mathematics and Statistics, University of Northumbria at Newcastle, Ellison Building, Newcastle upon Tyne, NE1 8ST, UK

We have considered two compilations of angular-size/redshift data for ultra-compact radio sources, due to Kellerman (1993) and Gurvits (1994) respectively, and a family of homogeneous isotropic universes with two degrees of freedom, represented by pressure-free matter and vacuum-energy. Kellerman's results seem to support the canonical model $q_{0}=1 / 2, \Omega_{0}=1$; the question posed here is: can we produce significant deceleration without dark matter?

The usual cosmological parameters are related by the dimensionless versions of Friedmann's equations

$$
q_{0}+\Lambda_{0}=\Omega_{0} / 2
$$

and

$$
\Omega_{0}+\Lambda_{0}=1+K_{0}
$$

If we believe that $q_{0}$ is large, equations (1) and (2) allow a clear alternative to large $\Omega_{0}$, namely $\Omega_{0} \sim 0, \Lambda_{0} \sim-q_{0}$, and $K_{0} \sim-1-q_{0}<0$, that is a violently decelerating open Universe, with dynamics dominated by a large negative cosmological constant. It turns out that the angular-size/redshift relationship is particularly sensitive to these parameters. In the limit $\Omega_{0}=0$ exactly there is an analytical expression giving the corresponding angular-diameter distance $d_{A}\left(q_{0}, z\right)$ (Jackson 1992), which is

$$
d_{A}=\frac{1}{q_{0} H_{0}}\left\{\left[1+q_{0}-q_{0}(1+z)^{-2}\right]^{1 / 2}-1\right\}
$$

Figure 1 compares the corresponding angular-size/redshift curves (labelled by values of $q_{0}$ ) with Kellerman's data, comprising 79 high-luminosity sources in 7 redshift bins, assuming a fixed linear size of $20.5 h^{-1}$ parsecs $\left(h=1 \Rightarrow H_{0}=100 \mathrm{~km} \mathrm{~s}^{-1} \mathrm{Mpc}^{-1}\right)$. Clearly there are curves which are compatible with the data, if we are prepared to contemplate values of $q_{0} \geq 3$. At this stage we make no claim that these vacuum-dominated universes are uniquely favoured by the data; the point is that there is in this context an acceptable model which invokes neither dark matter nor evolutionary effects. The crucial difference between these models and the canonical matter-dominated one is qualitative; in the latter case a pronounced increase in angular size is expected, beyond $z=1.25$, whereas in our models such behaviour is absent, corresponding to an asymptotic value $d_{A} \rightarrow\left(q_{0} H_{0}\right)^{-1}\left[\left(1+q_{0}\right)^{1 / 2}-1\right]$ as $z \rightarrow \infty$.

Tentatively, we can make a more positive statement than this. Very recently a large data compilation has appeared (Gurvits 1994), comprising 270 ultra-compact high-luminosity sources in 12 redshift bins in the range $0.5 \leq z \leq 3.8$. It is difficult to combine the two samples, as they use different definitions of size and correspond to different frequencies (5 Ghz and 2.3 Ghz respectively); nevertheless, we have attempted to do just this. Gurvits notes a reasonably systematic difference between his sizes and Kellerman's, the latter being 
larger than the former by a factor of about 3 over the high-redshift flat part of each diagram. Figure 2 shows a composite sample, comprising Kellerman's low-redshift points (at $z=0.047$ and $z=0.228$ ), and Gurvits' high-redshift points, scaled up in size by an appropriate fixed factor. At face value this diagram strongly favours the models proposed here, as there is no sign of the turn-up at high redshift expected in matter-dominated universes. A naive least-squares fit, giving free rein to $q_{0}$ and $\Omega_{0}$, and to the linear dimension $d$ characterising the sources, gives $q_{0}=8.34, \Omega_{0}=0.17$, and $d=15.5 h^{-1}$ parsecs. The theoretical curve (which is exact, i.e. does not use approximation (3)) shows a very gentle turn-up beyond $z \sim 4$, as the corresponding universe becomes matter-dominated.

One might expect the age problem associated with such high values of $q_{0}$ to be beyond salvation, but this class of models turns out to be remarkably forgiving in this context. This is illustrated by Figure 3, which plots age in Hubble units against $q_{0}$ for the limiting case $\Omega_{0}=0$ (solid line), to be compared with the standard matter-dominated case (dashed line); at a given value of $q_{0}$, ages are some $40 \%$ longer in the former case. The range $3 \leq q_{0} \leq 8$ requires $h$ to be in the range 0.3 to 0.4 , to give an age of $15 \times 10^{9}$ years, compared with $h=0.44$ in the canonical case.

\section{REFERENCES}

Gurvits L.I., 1994, ApJ, 425, 442

Kellerman K.I., 1993, Nature, 361, 134

Jackson J.C., 1992, QJRAS, 33, 17
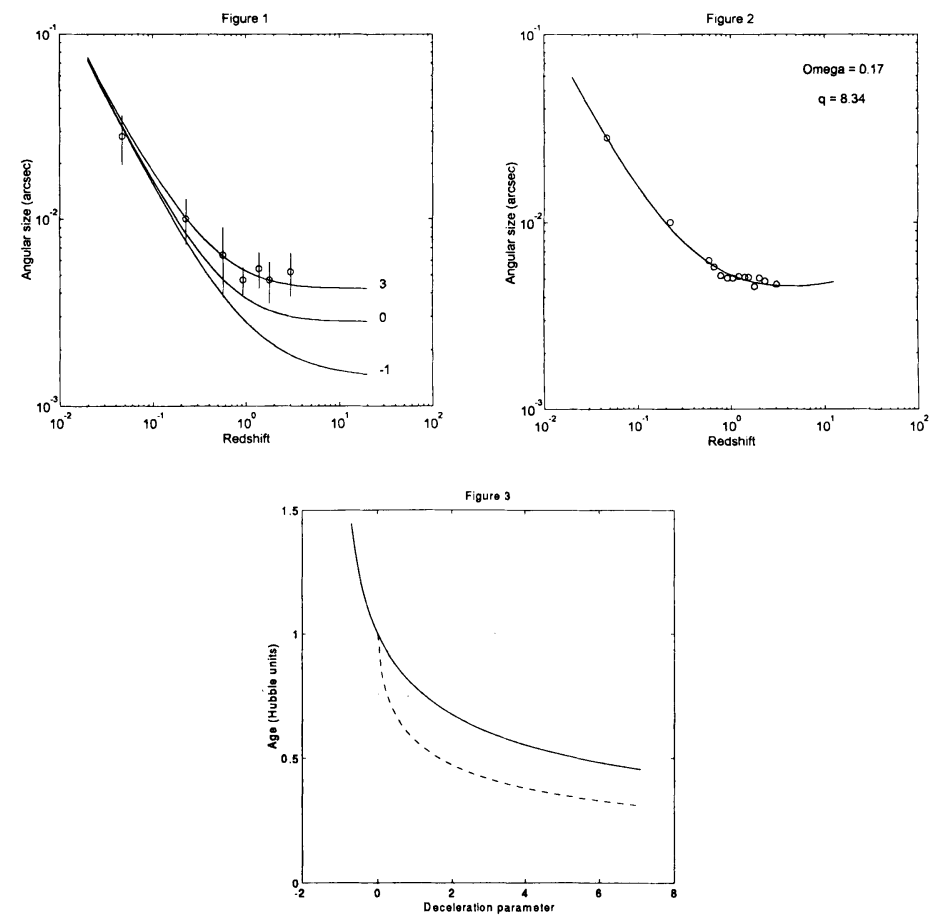